19 Revue d'histoire du XIXe siècle

Société d'histoire de la révolution de 1848 et des

révolutions du XIXe siècle

$55 \mid 2017$

Les écoles du peuple à l'ère des révolutions

$(1815-1880)$

« C'est du chinois ». À la recherche du chinois parisien (1814-1900)

"It's all Chinese to me". In search of Parisian Chinese (1814-1900)

"Das ist Chinesisch für mich". Auf der Suche nach dem Pariser Chinesisch

(1814-1900)

Clément Fabre

Q OpenEdition

Journals

Édition électronique

URL : http://journals.openedition.org/rh19/5342

DOI : 10.4000/rh19.5342

ISSN : $1777-5329$

Éditeur

La Société de 1848

Édition imprimée

Date de publication : 1 décembre 2017

Pagination : 171-188

ISSN : 1265-1354

Référence électronique

Clément Fabre, « «C'est du chinois ». À la recherche du chinois parisien (1814-1900) », Revue d'histoire

du XIXe siècle [En ligne], 55 | 2017, mis en ligne le 02 décembre 2019, consulté le 05 janvier 2021. URL http://journals.openedition.org/rh19/5342 ; DOI : https://doi.org/10.4000/rh19.5342

Tous droits réservés 


\section{CLÉMENT FABRE}

\section{"C'est du chinois".}

À la recherche du chinois parisien (1814-1900)'

«SGanarelle :

Veuillez me dire

Ce qu'il en est. - De quoi souffrez-vous notamment?

Quel mal ressentez-vous?

LUCINDE :

Han, hi, hon, han.

SGanarelle :

Comment?

LuCINDE: Han, hi, hon, han, han, hi, hon.

Sganarelle :

LUCINDE :

Quoi?

SGANARELLE :

LuCinde: Han, hi, hon, han, ha!

Han, hi, han.

Qu'est-ce?

SGANARELLE :

Y perdre mon latin. $»^{2}$

C'est du chinois, je confesse

L'expression "c'est du chinois", attestée dès la fin du XVIII siècle $^{3}$ et largement utilisée par les auteurs français tout au long du XIX ${ }^{\mathrm{e}}$, témoigne de la difficulté qu'il y a à saisir ce que recouvre à cette époque la catégorie de «langue chinoise». Par cette expression idiomatique, tout propos incompréhensible est intégré, du fait même de son incompréhensibilité, à cette catégorie qui englobe ainsi les objets les plus divers en fonction des contextes dans lesquels elle est utilisée. Cette expression attire donc l'attention sur l'historicité de la catégorie, et met en garde contre la tentation de plaquer artificielle-

1. Cet article est tiré d'un mémoire de master 2 lauréat du Prix d'histoire du XIXe siècle 2016 : Clément Fabre, Idéogrammes, caquetages et ornements. Paris et la langue chinoise (1814-1900), mémoire de master 2 sous la direction de MM. Hugues Tertrais et Pierre Singaravélou, Université Paris 1 Panthéon-Sorbonne, Centre d'Histoire de l'Asie Contemporaine, 2015. Version en ligne :

https://www.academia.edu/27681177/Id\%C3\%A9ogrammes_caquetages_et_ornements._Paris_ et_la_langue_chinoise_1814-1900

2. Émile Deschamps, "Le Médecin malgré lui (mis en vers)", in Euvres complètes. Théâtre, Deuxième Partie, Paris, Alphonse Lemerre, 1874.

3. Selon l'entrée "Chinois" du Dictionnaire culturel en langue française (Paris, Dictionnaires Le Robert, 2005), l'expression «C'est du chinois» est attestée depuis 1790. 
ment sur le chinois parisien du XIX ${ }^{\mathrm{e}}$ siècle nos conceptions contemporaines de l'idiome du Céleste Empire.

La réaction de Lucinde face à Sganarelle nous rappelle également que c'est pour rendre compte d'expériences sensibles que les acteurs parisiens $\mathrm{du}$ XIX ${ }^{\mathrm{e}}$ siècle mobilisent cette catégorie. Qu'il les ait identifiés comme chinois ou qu'il cherche comme ici à souligner leur étrangeté, l'individu qui désigne des sonorités ou des caractères comme relevant de la langue chinoise cherche par là à retraduire l'expérience qu'il en a faite. Aussi cette expression nous invite-t-elle, au-delà d'une histoire des mots utilisés pour dire la langue chinoise ${ }^{4}$, à étudier la genèse de cette catégorie à partir des expériences sensibles dont elle entend rendre compte. Il s'agit ainsi, dans la lignée d'une histoire des sensibilités représentée notamment par Hervé Mazurel et Christophe Granger ${ }^{5}$, de saisir d'un même mouvement la perception et la conception de la langue chinoise - couple que nous choisissons ici de désigner comme "confrontation" à la langue chinoise ${ }^{6}$.

Cette approche nécessite de restituer un certain environnement sensoriel des Parisiens de l'époque (à savoir l'ensemble des échantillons de langue chinoise auxquels ils sont susceptibles d'être confrontés), leur sensibilité à son égard ainsi que les différentes représentations et savoirs dont il fait l'objet. Ce qui nous invite à envisager la question d'une multiplicité de chinois parisiens : les individus ne conçoivent en effet pas la langue chinoise de la même manière selon l'expérience qu'ils en ont eue et les discours relatifs à cet idiome auxquels ils ont été confrontés. Faire l'archéologie du chinois parisien du XIX ${ }^{\mathrm{e}}$ siècle implique donc de restituer les différentes définitions et les diverses extensions qu'a pu y recouvrir cette catégorie en fonction des contextes individuels, ainsi que d'identifier les groupes sociaux caractérisés par une même appréhension de la langue chinoise. Aussi faut-il naviguer entre deux approches, l'une etic et l'autre emic, de la langue chinoise. Pour restituer l'environnement sensoriel des Parisiens du XIX ${ }^{\mathrm{e}}$ siècle, il s'agit de traquer la présence, dans l'espace parisien, d'une langue chinoise définie objectivement comme l'ensemble des styles historiques de l'écriture chinoise et des dialectes qui leur sont associés - qu'ils servent à les lire où se retranscrivent au moyen de ces caractères. Inversement, l'étude des conceptions de cette langue implique d'en adopter une définition subjective englobant tout ce que les acteurs du temps ont cru devoir subsumer sous cette catégorie.

Le cadre choisi pour mener cette étude s'explique par la place éminente qu'occupe tout au long du XIX ${ }^{\mathrm{e}}$ siècle la capitale parisienne dans le champ

4. Telle l'histoire prônée en 1961 par Georges Duby : "Histoire des mentalités ", in Charles Samaran [dir.], L'Histoire et ses méthodes, Paris, Gallimard, «Pléiade», 1961, p. 952.

5. Vingtième Siècle. Revue d'histoire, 2014/3, n 123, spécial : "Histoire des sensibilités au $20^{\mathrm{e}}$ siècle».

6. Samir Boumediene, dans l'ouvrage qu'il consacre au rapport des Européens aux plantes médicinales du Nouveau Monde à l'époque moderne, use ainsi à plusieurs reprises de cette idée de "confrontation" à l'altérité, qui articule la rencontre concrète et les conceptions de l'objet rencontré. Cf. Samir Boumediene, La colonisation du savoir, Paris, Les Éditions des mondes à faire, 2016. 
des études chinoises : de la création en 1814 de la chaire de langues et littératures chinoise et tartares-mandchoues de Jean-Pierre Abel-Rémusat, au Collège de France 7 , jusqu'à la fin du siècle ${ }^{8}$, Paris s'impose comme l'un des principaux foyers de la sinologie, comme une véritable Mecque des sinologues où convergent ouvrages de langue chinoise, sinologues et Chinois? Il importe de noter que l'espace parisien joue moins ici le rôle de catégorie analytique que d'observatoire privilégié, en raison de cette confluence. Parce que les acteurs qui la peuplent, la traversent et y publient ont plus de chance d'y rencontrer des échantillons de langue chinoise que sur le reste du territoire français, et parce qu'ils sont plus nombreux à s'y intéresser et à la prendre comme objet de leurs discours et de leurs études, la capitale permet de restituer les logiques qui guident ces confrontations à la langue chinoise, et notamment leur structuration sociale. La quantité d'expériences individuelles analysables permet en effet de dégager des régularités et d'identifier les espaces sociaux qui constituent des facteurs pertinents d'analyse. C'est ainsi que la rupture la plus féconde à étudier nous a semblé être, non pas celle qui oppose les sinologues au reste des Parisiens, mais celle qui oppose une population savante plus large, dont les membres ont un accès privilégié au chinois parisien et associent prioritairement la langue chinoise aux études sinologiques qu'elle suscite, à une population profane pour laquelle ces études jouent au mieux un rôle anecdotique, et n'orientent pas en tout cas la vision qu'ils ont de cet idiome, dont ils n'ont le plus souvent qu'une connaissance très rudimentaire.

C'est justement dans la dialectique qui oppose ces deux espaces sociaux que les conceptions parisiennes du chinois connaissent au XIX ${ }^{\mathrm{e}}$ siècle une inflexion marquée. La multiplication des possibilités de rencontre avec la langue chinoise et l'apparition d'un discours sinologique entendant traiter cette langue comme objet de science viennent contrebalancer la conception jusqu'alors majoritaire du chinois - une conception, inspirée des témoignages jésuites, qui faisait du chinois la langue la plus éloignée des idiomes connus et l'accablait de stéréotypes et de fantasmes (elle était ainsi tenue, tantôt pour une langue universelle, tantôt pour la langue primitive, toujours pour la langue la plus difficile qui soit ${ }^{10}$ ).

7. Arrêté de Louis XVIII donné au château des Tuileries, le 29 novembre 1814, Archives nationales (Arch. nat.), F/17/13553 : Collège de France. Affaires générales et collectives concernant les chaires, vacances et transformations. An XIII-1932. Dossier 1 : Chaires de chinois et de sanscrit. 1814-1815.

8. Les années 1898-1900 voient l'ouverture d'une chaire de chinois à Lyon ainsi que la mise en place de l'École française d'Extrême-Orient en Indochine, qui font perdre à Paris son monopole des études sinologiques françaises.

9. Hartmut Walravens, "Les recherches sur l'Extrême-Orient au début du XIXe siècle ou Paris, Mecque des orientalistes allemands", Revue germanique internationale, $\mathrm{n}^{\circ}$ 7, 2008 : «Itinéraires orientalistes", p. 35.

10. Viviane Alleton, L'écriture chinoise : Le défi de la modernité, Paris, Albin Michel, 2008; John DeFrancis, The Chinese Language : Fact and Fantasy, University of Hawaii Press, 1984; David Porter, Ideographia. The Chinese Cipher in Early Modern Europe, Stanford, Stanford University Press, 2001. 
Aussi s'agira-t-il de restituer le regard ${ }^{11}$ parisien sur la langue chinoise dans un double contexte de multiplication et de complexification des confrontations à cet idiome. Trois mouvements scanderont ce panorama du chinois parisien. Nous étudierons dans un premier temps les possibilités de rencontrer la langue chinoise dans le Paris du XIX ${ }^{\mathrm{e}}$ siècle : quels échantillons de cette langue y peut-on trouver? Quelle est leur accessibilité pour les différents groupes sociaux de la capitale? Quelle sensibilité, enfin, les Parisiens manifestent-ils à leur égard? La définition par la sinologie d'un chinois savant, conçu comme l'objet d'un savoir rationnel, occupera le deuxième temps de notre étude, laquelle se clora sur les représentations vulgaires qui entourent cet idiome et viennent brouiller les frontières de la catégorie élaborée par les savants.

\section{EXPÉRIENCES PARISIENNES DE LA LANGUE CHINOISE ET SENSIBILITÉS}

Outre les possibilités croissantes de voyage en Chine qui s'offrent aux Parisiens ${ }^{12}$ - sur lesquelles nous ne nous attarderons pas ici -, ces derniers sont susceptibles de rencontrer la langue chinoise à Paris même sous ses formes écrite et parlée.

La langue écrite est principalement représentée par les nombreux livres chinois que recèle la capitale. La collection de livres chinois de la Bibliothèque du Roi - la plus importante d'Europe - se développe à partir du $\mathrm{XVII}^{\mathrm{e}}$ siècle $^{13}$ et ne cesse de croître jusqu'à la fin de notre période. Au XIX ${ }^{\mathrm{e}}$ siècle, cette collection est alimentée par des dons individuels, par l'œuvre de correspondants officiels - notamment des interprètes au service du ministère des Affaires étrangères - et de missions scientifiques en Chine ${ }^{14}$, ou encore par l'absorption, en 1860, des collections chinoises des bibliothèques Mazarine, de l'Arsenal, Sainte-Geneviève et de la Sorbonne ${ }^{15}$. À côté de cette collection, la capitale française compte également la bibliothèque de l'École des langues orientales vivantes ${ }^{16}$ à partir de 1868, ainsi que les collections particulières de nombreux sinologues dont la correspondance privée permet d'appréhender les mécanismes de constitution. On trouve ainsi dans la correspondance de Julius Klaproth la trace de trocs réalisés avec diverses

11. Nous reprenons ici à notre compte l'approche de Rafael Mandressi, qui aborde le regard comme la manière dont l'individu met en forme le réel auquel il se retrouve confronté. $C f$. Rafael Mandressi, Le Regard de l'anatomiste, Paris, Seuil, 2003.

12. Avec les deux guerres de l'Opium (1839-1842 puis 1858-1861), les Français multiplient leurs implantations et leur présence diplomatique en Chine. Militaires, commerçants, diplomates et missionnaires sont ainsi de plus en plus nombreux à pouvoir accéder à la Chine.

13. Simone Balayé, La Bibliothèque Nationale des origines à 1800, Genève, Librairie Droz, 1988.

14. Clément Fabre, Idéogrammes, caquetages et ornements..., op. cit., p. 47-49.

15 Arrêté ministériel du 15 novembre 1860 cité dans Henry Martin, Histoire de la Bibliothèque de l'Arsenal, Paris, Librairie Plon, 1900, p. 579.

16. Elle fonctionne elle aussi grâce aux correspondants de l'École en Chine. 
bibliothèques européennes, d'achats d'ouvrages en Chine par l'intermédiaire de correspondants, ou encore d'échanges avec d'autres sinologues parisiens ${ }^{17}$.

Toutefois, d'autres supports plus inattendus mettent également les Parisiens aux prises avec les sinogrammes. On en trouve ainsi sur les nombreux objets d'art porteurs d'inscriptions qui sont achetés, collectionnés et exposés à Paris ${ }^{18}$. Les occasions de rencontrer la langue chinoise écrite se multiplient également lors des Expositions universelles. Les façades des pavillons chinois portent des inscriptions chinoises qui s'ancrent parfois durablement dans le paysage parisien - la pagode de l'Exposition de 1878 est ainsi offerte à la France et installée en 1880 dans le Bois de Boulogne ${ }^{19}$ - et l'on trouve dans «Le Courier de l'Exposition» du Monde Illustré la trace d'une rencontre surprenante avec la langue chinoise à l'occasion de l'Exposition de 1889. Le chemin de fer Decauville affiche, le long des contre-allées du Quai d'Orsay, des placards d'information pour prévenir les accidents, et les traduit en plusieurs langues pour toucher le public le plus large possible. Le public de l'Exposition se fait alors un jeu d'identifier ces langues, si bien "qu' on crut devoir ajouter de nouvelles affiches arabes, roumaines, grecques, turques, chinoises", et "qu'il devint urgent d'indiquer au bas, afin que nul n'en ignore, à quel idiome appartenaient les vocables bizarres qu'on venait de lire $»^{20}$.

Quant à la langue chinoise parlée, les Parisiens en font l'expérience grâce aux Chinois qui, tout au long du siècle, traversent et commencent à peupler la capitale. Avant le début de notre période, Paris avait déjà connu plusieurs visiteurs chinois, dont les plus connus sont Michel Chen Fo-Tsoung, qui rencontre en 1684 Louis XIV et lui récite des prières en chinois ${ }^{21}$, et Arcade Huang, lequel réside à Paris à partir de 1711 et devient "premier Interprète du Roi pour la langue chinoise $»^{22}$. Un véritable saut quantitatif est toutefois franchi au cours du XIX ${ }^{\mathrm{e}}$ siècle, à tel point que certains journaux en viennent sous le Second Empire à mentionner l'indifférence des Parisiens à l'égard de ces visiteurs qui semblent avoir perdu tout leur exotisme : "On

17. Hartmut Walravens, Julius Klaproth (1783-1835). Briefwechsel mit Gelehrten, großenteils aus dem Akademiarchiv in St. Petersburg, Wiesbaden, Harrassowitz Verlag, 2002 et Clément Fabre, op. cit., p. 53-55.

18. Paris compte ainsi plusieurs boutiques d'art chinois pour satisfaire un goût des chinoiseries hérité du XVIII e siècle et que vient renforcer l'afflux d'objets chinois consécutif au sac du palais d'Été. Le «Magasin de la porte chinoise», et les magasins de M. Leblanc sont mentionnés par la presse dès la fin des années 1820 , et la deuxième moitié du siècle voit apparaître les célèbres boutiques "À la porte chinoise» et "La Porte Chinoise». Mentionnons enfin, à la fin du siècle, les boutiques «Au Mikado" et le "Palais d'été", ainsi que l'hôtel Drouot où, tout au long du siècle, sont vendus aux enchères et exposés des objets chinois.

19. L. Dutartre, "Conseil municipal - Séance du 29 avril», Le Figaro, 30 avril 1880, p. 3.

20. G. Lenôtre, "Courrier de l'Exposition", Le Monde Illustré, tome 65, n 1698, 12 octobre 1889 , p. 227. On retrouve également la trace de cette anecdote dans Le Figaro: Georges Grison, «Courrier de l'Exposition», Le Figaro, 12 septembre 1889, p. 2.

21. J. Dehergne S. J., "Voyageurs chinois venus à Paris au temps de la marine à voiles et l'influence de la Chine sur la littérature française du XVIII" siècle", Monumenta Serica, volume 23, 1964.

22. Cécile Leung, Etienne Fourmont (1683-1745). Oriental and Chinese languages in eighteenthcentury France, Leuven, Leuven University Press, 2002. 
s'efforce de faire quelque bruit autour de l'ambassade chinoise. Mais cela ne prend guère. Le Parisien, voyez-vous, commence à se blaser de ces missions devenues aussi par trop fréquentes ${ }^{23}$.» Nous avons identifié trois catégories principales de Chinois parisiens : les visiteurs occasionnels, la colonie chinoise de Paris $^{24}$ et les diplomates chinois qui s'installent plus ou moins durablement dans la capitale et constituent ainsi une catégorie intermédiaire $^{25}$. Nous ne dresserons pas ici l'inventaire détaillé de tous les voyageurs occasionnels ${ }^{26}$, mais deux types principaux doivent toutefois être identifiés parmi eux : les Chinois venus à l'occasion des Expositions universelles - en 1867, «deux ravissantes Chinoises ${ }^{27}$ servent le thé dans le restaurant chinois de l'Exposition, et les architectes, peintres, sculpteurs et ouvriers qui réalisent les palais chinois retiennent l'attention de la presse en $1878^{28}$ comme en $1900^{29}$ - et les troupes d'artistes chinois ${ }^{30}$. Il est plus important d'étudier en détail la colonie chinoise qui se développe à Paris au long du siècle, et qui retient suffisamment souvent l'attention de la presse pour que l'on puisse en appréhender l'importance et la constitution. Alors qu'un article du Journal asiatique affirme en 1823 que seuls trois Chinois se sont installés durablement à Paris depuis la Révolution ${ }^{31}$, cette communauté embryonnaire croît rapidement après la réouverture de la Chine. On sait ainsi que «la petite colonie d'hommes jaunes $»^{32}$, qui compte "cinq ou six sujets $»^{33}$ en 1872 , passe de sept Chinois en $1875^{34}$ à 50 en 1894 : « 3 vieillards, 24 jeunes gens, 13 femmes et 10 enfants ${ }^{35}$; et deux articles de $1891^{36}$ et $1894^{37}$ affirment qu'elle peut être décomposée en "gens de marque», en "marchands" et en "employés» de commerce ou de maison. Sans dresser le portrait de chacun d'entre eux, il importe d'identifier certains Chinois qui jouent un rôle particulièrement important dans l'accès des Parisiens à la langue chinoise. Lieou-Sieou Tchang, ramené à Paris en $1846^{38}$ par l'interprète Joseph-Marie

23. John Stick, "Coups de plumeau», Le Tintamarre, $28^{e}$ année, 7 février 1869, p. 2.

24. Ce sont les journaux parisiens eux-mêmes qui, à plusieurs reprises au cours du siècle, évoquent en ces termes l'embryon de communauté chinoise que connaît la capitale française.

25. Clément Fabre, op. cit., p. 59 sq.

26. Ibidem.

27. Georges Maillard, «Hier - Aujourd'hui - Demain», Le Figaro, 8 décembre 1866, p. 2.

28. Jean de Paris, "Nouvelles diverses», Le Figaro, 8 janvier 1878, p. 3.

29. Jules Huret, "Artistes exotiques", Le Figaro, 9 avril 1900, p. 2.

30. Ces troupes ont été étudiées en détails par Shih-Lung Lo, La Chine dans le théatre français du XIXe siècle, thèse de doctorat, Paris, Université de la Sorbonne Nouvelle, 2011.

31. L. B. "Historique de l'instruction du Chinois qui a été présenté au Roi, le 8 octobre 1821, par M. Philibert, capitaine de vaisseau, et député de l'Ile de Bourbon à Paris; par $\mathrm{M}^{\mathrm{me}}$ Celliez née Clle de Rosis, Blois, 1822, 20 pages in $4^{\circ}$ ", Journal asiatique, $n^{\circ} 2,1823$, p. 48.

32. Arsène Alexandre, "Au Jour le Jour - Nos Chinois", Le Figaro, 16 mars 1891, p. 1.

33. Punch, "Nos informations», Le Figaro, 30 décembre 1872, p. 3.

34. Gaston Vassy, «Informations. La colonie chinoise de Paris», Le Figaro, 3 février 1875, p. 2.

35. Marcel Hutin, "Au Jour le Jour - Japonais et Chinois à Paris», Le Figaro, 17 août 1894, p. 2.

36. Arsène Alexandre, op. cit.

37. Marcel Hutin, op. cit.

38. Archives de la Courneuve, Personnel $1^{\text {ère }}$ série, dossier 393QO/710 : Callery Joseph Marie, lettre de Callery au ministre des Affaires étrangères (Paris, $1^{\text {er }}$ octobre 1846). 
Callery, sert ainsi de répétiteur chinois aux Langues $\mathrm{O}^{\prime 39}$, mais c'est principalement Tin-Tun-Ling, "le mandarin de Théophile Gautier " ${ }^{40}$, qui s'impose comme l'archétype du Chinois parisien. Arrivé depuis Macao à Paris en 1861 comme secrétaire de Callery, il devient à tel point une célébrité parisienne que Le Figaro peut écrire en 1868 que «tout Paris connaît Tin-Tun-Ling, le Chinois de Th. Gautier » ${ }^{41}$. Son rôle de passeur entre les Parisiens et la langue chinoise tient particulièrement à la place de répétiteur qu'il occupe auprès du marquis d'Hervey de Saint-Denys pour ses cours au Collège de France et à l'École spéciale des langues orientales vivantes, ainsi qu'aux cours qu'il dispense dans la capitale, notamment auprès de Judith Gautier, fille du célèbre poète du Parnasse ${ }^{42}$. Ce rôle d'intermédiaire est également endossé par certains des diplomates qui - à partir de 1866 puis surtout avec l'établissement de légations régulières en $1876^{43}$ - investissent la capitale française. À partir de 1887, les répétiteurs indigènes pour la langue chinoise aux Langues O' - qui existent depuis 1874 - sont ainsi recrutés au sein du personnel diplomatique chinois ${ }^{44}$.

À côté de ces échantillons "purs» de langue chinoise, les Parisiens peuvent également accéder à la langue chinoise grâce à divers outils européens destinés à réduire l'écart séparant cette langue du public français. Outre les différents cours de chinois que compte la capitale ${ }^{45}$, Paris voit ainsi se multiplier les outils d'apprentissage du chinois écrit : dictionnaires, manuels, grammaires ou encore traductions bilingues ${ }^{46}$. La multiplication de ces outils tient notamment aux progrès notoires de l'impression chinoise au cours du siècle ${ }^{47}$. L'Imprimerie nationale recèle dès le début du XIX ${ }^{e}$ siècle un corps de caractères chinois gravés à partir de 1720 sous la supervision du protosinologue Étienne Fourmont, mais les difficultés que présente l'usage de ces caractères en bois, endommagés et d'une grosseur démesurée, poussent les sinologues à multiplier tout au long du siècle les initiatives pour faciliter les

39. L'École des langues orientales vivantes est connue sous ce surnom tout au long du siècle.

40. Parisis, «La vie parisienne - La Chine à Paris», Le Figaro, 9 mai 1884, p. 1.

41. Georges Maillard, «Hier - Aujourd'hui - Demain», Le Figaro, 7 mars 1868, p. 1.

42. William Leonard Schwartz, The imaginative interpretation of the Far East in modern French literature, 1800-1925, Paris, librairie ancienne Honoré Champion, 1927, p. 21-23.

43. André Lévy, Nouvelles lettres édifiantes et curieuses d'Extrême-Occident par des voyageurs lettrés chinois à la Belle Époque (1866-1906), Paris, Seghers, 1986.

44. Lettre de l'administrateur de l'École au ministre de l'Instruction publique (Paris, 12 janvier 1887). Cité dans Laurent Galy, "Les "répétiteurs indigènes pour la langue chinoise" (1873-1925)", in Marie-Claire Bergère et Angel Pino [dir.], Un siècle d'enseignement du chinois à l'école des langues orientales, 1840-1945, Paris, L'Asiathèque, 1996, p 295.

45. Après l'ouverture de la chaire du Collège de France, Paris compte un cours de chinois moderne aux Langues $\mathrm{O}$ 'à partir de 1841 - officialisé en 1843, mais également d'autres enseignements plus anecdotiques, tels le cours libre de chinois assuré de 1869 à 1871 par le comte de Kleczkowski (futur professeur aux Langues O’) le cours de chinois épistolaire ouvert en 1891 par Léon de Rosny à l'École coloniale, ou encore le cours de chinois dispensé à la fin du siècle à l'Institut catholique de Paris, cf. Clément Fabre, op. cit., p. 90-91.

46. Clément Fabre, op. cit., p. 85 sq.

47. Georg Lehner, Der Druck chinesischer Zeichen in Europa. Entwicklungen im 19. Jahrhundert, Wiesbaden, Harrassowitz Verlag, 2004. 
impressions en chinois. Rémusat en 1817 et Klaproth en 1830 supervisent ainsi la création de caractères chinois. Toutefois, la création la plus célèbre en ce domaine demeure celle entreprise à partir des années 1830 par Guillaume Pauthier et Marcellin Legrand - graveur de l'Imprimerie royale - qui réalisent un corps complet de types chinois décomposables en acier. Parallèlement, certains optent pour l'achat de caractères réalisés en Chine - Stanislas Julien dès 1841, la Société Asiatique dans les années 1850, ou encore Paul Perny en 1873. Enfin, certains auteurs préfèrent à la typographie l'usage de l'impression lithographique, qui connaît un véritable essor de 1815 aux années 1830, avant que la typographie ne l'emporte finalement. Notons que la difficulté de ces techniques d'impression restreint largement le nombre d'imprimeurs qui s'y livrent, ainsi que le nombre de maisons d'édition proposant des ouvrages chinois - outre l'Imprimerie nationale, on compte ainsi la maison Dondey-Dupré puis les éditeurs Firmin-Didot, Ernest Leroux et Maisonneuve et $\mathrm{C}^{\mathrm{ie} 48}$.

Étudier les expériences parisiennes du chinois parisien implique également de restituer l'accès des différentes catégories de la population parisienne à ces échantillons de langue chinoise ${ }^{49}$. Or, si l'accès aux voyageurs du Céleste Empire et à la colonie chinoise semble connaître une véritable démocratisation au cours du siècle - dont témoigne l'indifférence croissante des Parisiens à leur égard -, l'existence de cours de chinois à Paris est largement ignorée par les habitants de la capitale, et les manuscrits des différentes collections ne sont accessibles qu'aux seuls sinisants. Se dégage ainsi l'image d'une société parisienne à deux vitesses, partagée entre un Paris savant qui seul a accès à l'ensemble des échantillons parisiens de langue chinoise, et un Paris profane qui n'a accès qu'à des bribes de chinois parlé et à de rares sinogrammes glanés sur les façades de certains établissements, sur des affiches occasionnelles ou sur des objets d'art.

Quelques rares sources permettent enfin de saisir, dans une certaine mesure, la sensibilité des Parisiens à l'égard des échantillons de langue chinoise qu'ils viennent à rencontrer. À l'occasion du procès intenté en 1875 à Tin-Tun-Ling pour polygamie, la manière dont Le Figaro justifie la décision du tribunal d'empêcher le célèbre Chinois d'assurer lui-même sa défense nous en offre un premier indice : "on a pensé que son jargon franco-chinois éveillerait une hilarité peu compatible avec la gravité de la circonstance ${ }^{50}$. Les Parisiens semblent ainsi percevoir au premier abord le chinois parlé comme une langue éminemment étrange et ridicule, idée que viennent conforter de multiples témoignages. Les Frères Goncourt évoquent en 1863 le «caquetage exotique» des filles de Théophile Gautier récitant les leçons de Tin-Tun-

48. Clément Fabre, op. cit., p. 91-92.

49. Idem, p. 95 et sq.

50. Gaston Vassy, «Informations», Le Figaro, 11 juin 1875, p. 2. 
Ling $^{51}$, et les deux spectacles que donne ce dernier en 1874 entraînent un déferlement de commentaires hilares. Gaston Vassy souligne la bizarrerie des "syllabes nasales et pétillantes "52 déclamées par Tin, tandis que Jules Préval déplore que la récitation de ses poèmes soit suivie d'une traduction française : "Si l'on comprend, ça ne sera plus aussi drôle " ${ }^{33}$. Notons que cette impression de ridicule touche jusqu'aux sinologues : Rémusat évoque ainsi en 1815 les "prononciations bizarres " ${ }^{54}$ du chinois et reconnaît en 1826 que les études chinoises pâtissent du ridicule qui frappe les mots chinois, lesquels "sont particulièrement désagréables et difficiles à prononcer " ${ }^{55}$. Quant aux sinogrammes, ils semblent être généralement perçus comme des dessins plus que comme des signes porteurs de sens. Les catalogues des ventes publiques d'objets d'art chinois livrent ainsi des descriptions particulièrement éloquentes d'inscriptions chinoises, parmi lesquelles nous pouvons citer ce «vase en or massif, à ornements, fleurs, feuillages et inscriptions très-finement ciselés en relief " ${ }^{56}$, ou encore cette «très belle applique [...] enrichie de feuilles, branchages, fleurs et caractères $"$ " ${ }^{57}$. Certaines caricatures véhiculent enfin la même sensibilité à la langue chinoise écrite, telle. cette mise en scène d'un Parisien observant avec étonnement les caractères qu'il voit tracer sous ses yeux $^{58}$ :

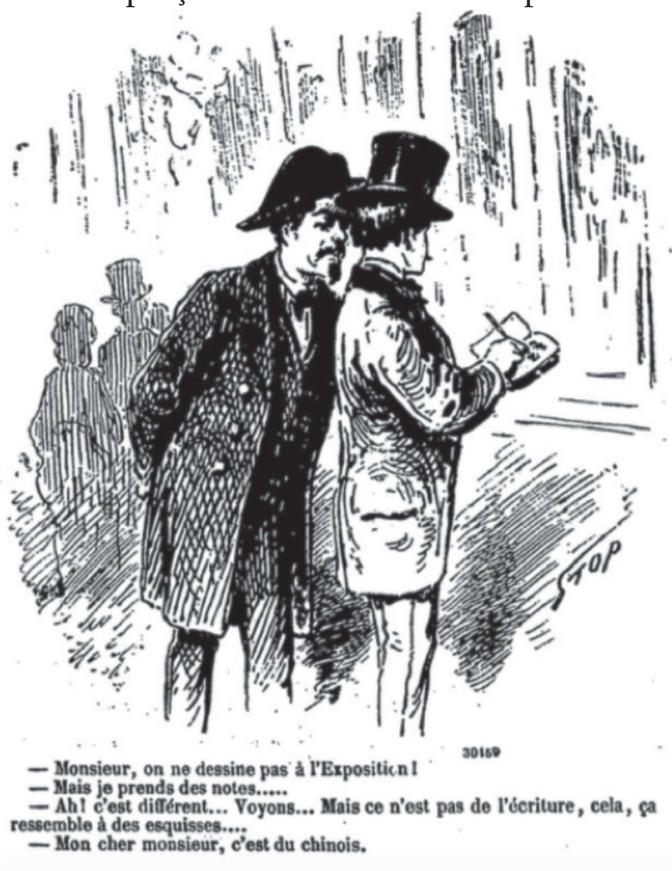

51. Journal des Goncourt: Mémoires de la vie littéraire, Paris, Bibliothèque Charpentier, 1891, consulté en ligne : vendredi 17 juillet. URL : http://fr.wikisource.org/wiki/Journal_des_Goncourt.

52. Gaston Vassy, «Informations. La Journée», Le Figaro, 7 juillet 1874, p. 3.

53. Jules Préval, "Courrier des Théâtres", Le Figaro, 21 mai 1874, p. 3.

54. Jean-Pierre Abel-Rémusat, "Discours prononcé à l'ouverture du cours de langue et de littérature chinoises, au Collège Royal, le 16 janvier 1815; sur l'origine, les progrès et l'utilité de l'étude du chinois en Europe", in Mélanges asiatiques, ou choix de morceaux de critique et de mémoires relatifs aux religions, aux sciences, aux coutumes, à l'histoire et à la géographie des nations orientales, tome 2, Librairie orientale de Dondey-Dupré père et fils, 1826, p. 12.

55. Jean-Pierre Abel-Rémusat, Iu-kiao-li, ou les deux Cousines; Roman Chinois précédé d'une préface où se trouve un parallèle des romans de la Chine et de ceux de l'Europe, Paris, Moutardier, 1826, consulté sur Internet. URL : www.chineancienne.fr

56. Anonyme, "Vente publique aux enchères d'objets d'art et de curiosité de la Chine, provenant du Palais Yuen- Ming-Yuen, faite à Paris les 30 et 31 janvier 1862 ", Journal des amateurs d'objets d'art et de curiosité, 1862, tome 9.

57. Anonyme, "Vente publique aux enchères d'objets d'art et de curiosité de la Chine, faite à l'hôtel des commissaires-priseurs de Paris, le 23 novembre 1863 (M. Manheim, expert) ", Journal des amateurs d'objets d'art et de curiosité, 1864, tome 10, objets $\mathrm{n}^{\circ} 82,93$ et 95 .

58. Stop, "Le Salon de $1872 »$, Le Journal Amusant. Journal illustré, Journal d'images, journal 
Le Paris du XIX ${ }^{\mathrm{e}}$ siècle apparaît ainsi comme le théâtre d'expériences de la langue chinoise qui dépendent des échantillons de cette langue présents dans la capitale, qui varient selon les groupes sociaux et dans lesquelles transparaît l'étonnement - souvent moqueur, parfois admiratif - avec lequel les Parisiens perçoivent cet idiome. La capitale française accueille toutefois concomitamment l'élaboration d'un discours sinologique qui tente, par-delà cette première impression d'étrangeté, de définir scientifiquement cette langue.

\section{LE CHINOIS SAVANT}

La sinologie moderne qui prend naissance avec l'ouverture de la chaire de Rémusat se fonde avant tout sur le rejet des définitions du chinois héritées du XVIII ${ }^{e}$ siècle. Contre les discours essentialistes qui recherchaient dans la langue chinoise une langue universelle, ou encore la langue originelle transmise par un Noé identifié à l'empereur mythique Huangdi, Rémusat affirme dans un geste fondateur que la langue chinoise est une langue comme une autre et doit être étudiée comme un simple moyen de communication.

Comment donc, après avoir rejeté ces conceptions schématiques, la sinologie définit-elle la langue chinoise? Elle s'évertue à la définir à la fois dans son principe et par ses limites. Pour ce qui est du principe qui régit la langue chinoise, Rémusat ouvre tout d'abord une brèche dans la définition du chinois comme essentiellement monosyllabique et idéographique : tout au long du XIX ${ }^{\mathrm{e}}$ siècle, qu'ils se rallient finalement à l'idée du monosyllabisme et du caractère idéographique du chinois ou qu'ils la rejettent, les sinologues ne peuvent plus se dispenser de la discuter ${ }^{59}$. Reprenant les travaux réalisés en Chine par le Père jésuite de Prémare, le fondateur de la sinologie moderne voit dans la position des mots l'essence même du système de communication original que constitue le chinois - et cette thèse est largement reprise par ses successeurs jusqu’à la fin du siècle.

L'apport le plus important de la sinologie dans la perspective qui nous intéresse ici consiste toutefois dans sa tentative de définir les frontières de la langue chinoise : quels dialectes, quelles écritures relèvent du chinois ?60 La principale difficulté attachée à cette délimitation tient à l'idée, héritée du XVIII ${ }^{\mathrm{e}}$ siècle, selon laquelle coexistent plusieurs langues chinoises : une langue écrite et une multitude de langues parlées. Si les jésuites et les protosinologues accordent une place à part à la langue mandarine parmi ces langues parlées, toutes apparaissent comme autant de dialectes pouvant être retranscrits au moyen de la langue écrite. Aussi cette conception - qui jus-

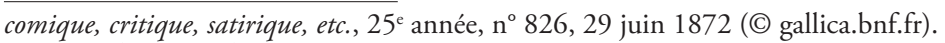

59. Clément Fabre, op. cit., p. 135-142.

60. Idem, p. 29-37. 
tifie le fantasme d'un chinois-langue universelle - conduit-elle les auteurs à assimiler les diverses langues de l'aire sinisée à des dialectes chinois, au prétexte que le Japon, la Corée ou encore l'Annam ont largement emprunté à la Chine ses caractères d'écriture.

Ces oppositions entre langue écrite et langue parlée, entre langue savante et langue vulgaire, sont reprises au XIX ${ }^{\mathrm{e}}$ siècle, mais complexifiées par la prise en compte d'une nouvelle distinction entre langue ancienne et langue moderne - ces multiples lignes de fracture se combinant différemment selon les auteurs. Contre la vision du XVIII ${ }^{\mathrm{e}}$ siècle, Rémusat propose ainsi l'idée d'un continuum de styles chinois allant, au fil d'une évolution historique, d'une langue antique purement écrite - le kou-wen - à la langue parlée de son temps, ou kouan-hoa. Cette conception est toutefois vite remise en cause, car tributaire des échantillons de langue chinoise auxquels Rémusat a pu avoir accès : n'ayant jamais mis les pieds en Chine, il ne peut aborder la langue parlée en Chine à l'époque qu'à travers des romans vieux de plus d'un siècle censés retranscrire cette langue parlée, mais qui en different en fait considérablement. Aussi les voyageurs européens qui investissent en nombre la Chine à partir de 1839 ont-ils tôt fait de repousser les conceptions rémusiennes pour remettre en avant la distinction primordiale entre chinois écrit et chinois parlé. Le XIX ${ }^{\mathrm{e}}$ siècle voit ainsi se complexifier la conception des lignes de fracture qui parcourent la langue chinoise.

La multiplication des voyages en Chine met également les Européens aux prises avec des formes linguistiques de plus en plus nombreuses dont il importe de déterminer si elles relèvent ou non du chinois. L'intégration des patois parlés en Chine à la langue chinoise commence ainsi à faire l'objet d'âpres débats. Le cantonais en offre le meilleur exemple : alors que Callery le considère comme l'un des trois principaux dialectes de la Chine - à côté du mandarin et du fokiénois - ${ }^{61}$, le Commandant Lagarrue affirme en 1900 que, loin d'être un simple dialecte du chinois, le cantonais constitue une langue à part entière ${ }^{62}$. Cette différence s'explique par les perspectives divergentes avec lesquelles les auteurs abordent le chinois : alors que Callery, en qualité d'interprète, se soucie uniquement de restituer un savoir pratique sur les diverses prononciations utilisées en Chine pour lire les caractères chinois, des auteurs comme le Commandant Lagarrue s'intéressent, dans la lignée des linguistes, à des familles de langues parlées qu'ils tâchent d'appréhender indépendamment des caractères qui servent à les transcrire. Une fois dissipés les fantasmes entourant l'écriture chinoise, la Chine et l'Asie prennent ainsi progressivement le même visage que l'Europe : celui d'une diversité de langues unifiées par l'usage du latin.

61. Joseph-Marie Callery, Dictionnaire encyclopédique de la langue chinoise, Paris, Typographie de Firmin Didot Frères, 1842, p. XI.

62. Commandant Lagarrue, Éléments de Langue chinoise dialecte cantonais Notation Quôc Ngu. À l'usage des Officiers, Fonctionnaires et Colons, Paris, Ernest Leroux, 1900, p. I. 
La question des autres langues asiatiques et celle, surtout, des langues anciennes de la Chine montrent toutefois que la définition du chinois par l'écriture chinoise demeure largement dominante. Même si Rémusat récuse l'idée selon laquelle les langues des Tonquinois, des Cochinchinois et des Japonais ne seraient qu'autant de dialectes du chinois ${ }^{63}$, Léon de Rosny se permet en 1856 de comparer le "dialecte de la Corée» au dialecte du Fokien $^{64}$. Plus révélateur encore : lorsque Terrien de Lacouperie entreprend d'étudier les langues parlées en Chine avant l'introduction de l'écriture chinoise, il refuse de considérer ces langues comme relevant de la langue chinoise, comme l'exprime clairement le titre de son ouvrage : Les Langues de la Chine avant les Chinois ${ }^{65}$. Malgré les multiples nuances et complexifications apportées tout au long du siècle, la sinologie continue donc de définir avant tout la langue chinoise par l'usage des caractères chinois : est chinoise toute langue qui s'écrit au moyen de sinogrammes.

Si la sinologie élabore ainsi une conception du chinois comme un simple système de communication, identifiable par un certain emploi de la position des mots et par l'usage d'une écriture originale, encore faut-il déterminer la diffusion que connaît ce chinois savant dans le Paris du XIX ${ }^{\mathrm{e}}$ siècle $^{66}$. Le discours sinologique use de plusieurs vecteurs pour toucher une population parisienne plus vaste que les seuls sinisants. Outre les encyclopédies et dictionnaires étudiés par Georg Lehner ${ }^{67}$, deux canaux de diffusion sont principalement utilisés : les revues de vulgarisation ${ }^{68}$ et les traductions de littérature chinoise réalisées par les sinologues. Ces dernières prétendent en effet offrir la possibilité d'une double lecture, le texte même pouvant être apprécié par n'importe quel lecteur tandis que l'appareil critique offre au sinisant l'instrument d'une lecture sinologique. Les sinologues mettent ainsi dans les mains d'un public profane des ouvrages truffés d'informations sur la langue chinoise, et les préfaces témoignent tout particulièrement de leur désir de vulgariser un certain nombre de connaissances sinologiques. Dans la préface de ses Poésies de l'époque Thang, le marquis d'Hervey de Saint-Denys, titulaire de la chaire du Collège de France, expose ainsi les diverses thèses sinologiques sur les différences entre langue écrite et langue parlée, le monosyllabisme et le caractère idéographique du chinois, et diffuse ainsi une image de cette langue qui tranche avec celle véhiculée par les revues de vulgarisation et les encyclopédies. Les articles de ces dernières sont en effet rarement l'œuvre des

63. Jean-Pierre Abel-Rémusat, "Sur les Nouvelles Lettres Édifiantes», in Mélanges asiatiques..., op. cit., tome 1, 1825, p. 63.

64. Léon de Rosny, Introduction à l'étude de la langue japonaise, Paris, Maisonneuve et Cie, 1856, p. 4.

65. Albert Étienne Jean-Baptiste Terrien de Lacouperie, Les Langues de la Chine avant les Chinois - Recherches sur les langues des populations aborigènes et immigrantes, l'arrivée des Chinois, leur extension progressive dans la Chine propre et les sources de leur civilisation, Paris, Ernest Leroux, 1888.

66. Clément Fabre, op. cit., p. 299-314.

67. Georg Lehner, China in european encyclopaedias, 1700-1850, Leiden, Boston, Brill, 2011.

68. Nous avons étudié Le Magasin Pittoresque et le Journal des voyages. 
sinologues les plus reconnus : lorsque les articles ne sont pas anonymes, ce sont le plus souvent des sinologues de terrain - tels les interprètes Callery ou Camille Imbault-Huart -, ou encore des sinologues rejetés par les institutions parisiennes - tel Guillaume Pauthier - qui les signent, et la plupart des articles cherchent à exploiter la curiosité du public pour cet idiome étrange plus qu'à l'informer à son propos. Ces articles regorgent ainsi d'exposés sur les «bizarreries de la langue chinoise ${ }^{69}$, et notamment de son écriture, reléguée comme au XVIII siècle au rang de curiosité hiéroglyphique.

On voit ainsi le chinois savant se dissoudre, malgré les efforts de diffusion des sinologues, dans des instruments de vulgarisation qu'ils contrôlent insuffisamment, et où point à nouveau l'image d'un chinois conçu comme paradigme de l'altérité. Aussi importe-t-il de sonder les conceptions de la langue chinoise qui transparaissent des discours profanes - depuis la presse grand public jusqu'à la littérature - pour examiner la pertinence d'un modèle diffusionniste appliqué au chinois parisien : dans quelle mesure le chinois profane reflète-t-il le chinois savant?

\section{LE CHINOIS PROFANE}

La plupart des représentations véhiculées sur la langue chinoise dans le Paris profane traduisent bien moins une diffusion des conceptions sinologiques qu'un jeu sur la sensibilité - déjà esquissée - des Parisiens à la langue chinoise. Les images d'une langue chinoise ridicule et incompréhensible essaiment ainsi dans la presse comme dans la littérature.

Il est tout d'abord courant, pour les auteurs du XIX ${ }^{\mathrm{e}}$ siècle, de moduler à l'infini l'expression "c'est du chinois", la langue chinoise apparaissant ainsi comme l'une des manières les plus répandues - en concurrence, toutefois, avec d'autres langues rares - de signifier l'incompréhension. Dans Le Cousin Pons de Balzac, on peut ainsi lire après une tirade du personnage éponyme que " [l]a mère et la fille se regardaient comme si Pons leur eût parlé chinois » ${ }^{70}$ et Chiquita tient chez Théophile Gautier un langage "aussi inintelligible pour des Français que du haut allemand, de l'hébreu ou du chinois »71. Le même procédé sert parfois à souligner l'hermétisme des sentiments : Alexandre Dumas évoque "la grammaire chinoise de l'âme humaine »" et l'héroöne des Caprices de Marianne évoque en ces termes l'amour qui lui est voué : «Il faut croire que sa passion pour moi était quelque chose comme du chinois ou

69. G. R., "La langue chinoise», Journal des voyages et des aventures de terre et de mer, 1896, Second Semestre, p. 190.

70. Honoré de Balzac, Le Cousin Pons, [1847], Paris, Alexandre Houssiaux, 1855. Retranscrit dans la B.E.Q., consulté en ligne, p. 406.

71. Théophile Gautier, Le Capitaine Fracasse [1863], Paris, G. Charpentier et Cie, 1889. Retranscrit dans la B.E.Q., consulté en ligne, p. 689.

72. Alexandre Dumas, La Princesse Flora [1859], Paris, Michel Lévy Frères, 1871. Retranscrit dans la B.E.Q., consulté en ligne, p. 190. 
de l'arabe, puisqu'il lui fallait un interprète, et qu'elle ne pouvait s'expliquer toute seule $»^{73}$. Cet usage de la catégorie «langue chinoise» sert souvent enfin les besoins de la polémique. Citons comme seul exemple un mot célèbre de $\mathrm{M}^{\mathrm{me}}$ de Staël à propos du baron de Norvins : "Il y a deux langues que je ne comprends pas, le chinois et le Norvins $»^{74}$.

Autre indice du ridicule qui frappe cette langue : le théâtre invente couramment de pseudo-termes chinois, identifiables comme chinois uniquement en raison de leur bizarrerie. Ce procédé, utilisé dès le XVIII ${ }^{e}$ siècle $^{75}$, est largement repris au XIX ${ }^{\mathrm{e}}$, où il a été étudié par Shih-Lung $\mathrm{Lo}^{76}$. Dans la pièce La Mère Moreau, présentée au Palais-Royal en 1852, des clients jouent ainsi les Chinois en chantant «Ki, ka, ké, ko, ku, / Ba, bé, bi, bo, bu, / Ka, ka, / $\mathrm{Ki}$, ki, ri, ki, ki, ki, / Ka, ka, ka, pi, pi, / O li » ${ }^{77}$. De même, les Bouffes-Parisiens présentent en 1855 le Pékinois Ké-ki-ka-ko dans la pièce $B a-t a-\operatorname{clan}^{78}$, où un Quatuor chinois entame un chant sur les paroles "Maxalla chapallaxa" et "Rapataxa rafaxa " ${ }^{79}$ - notons que les auteurs ne prennent même pas la peine de choisir des syllabes ressemblant aux syllabes chinoises, tant leur étrangeté suffit à les identifier comme telles. On retrouve d'ailleurs le même procédé dans la littérature : Georges Sand parodie à son tour le chinois dans son roman Consuelo : La Comtesse de Rodolstadt, lorsqu'elle évoque une pièce dans laquelle l'héroïne doit déclamer les vers suivants :

"Ping, pang, tiong,

$H i$, han, hong

Tel était le refrain, qui était censé signifier, grâce à la puissance d'abréviation que possédait cette langue merveilleuse :

"Belle margrave, grande princesse, idole de tous les cœurs, régnez à jamais sur votre heureux époux et sur votre joyeux empire de Roswald en Moravie" ${ }^{\text {." }}$

De même, les caractères de chinois, lorsqu'ils apparaissent dans des illustrations, sont le plus souvent réduits à des "dessins bizarres" ${ }^{81}$.

73. Alfred de Musset, Les Caprices de Marianne [1833], Paris, Charpentier, 1851, p. 35-36.

74. Anonyme, "Caquets", Tintamarre, 30 juillet $1843-6$ août 1843 , p. 7.

75. Pierre Peyronnet, " “C'est pour rire..." ou la Chine sur le théâtre français au dix-huitième siècle», Dalhouse French Studies, volume 43, Summer 1998, p. 119-129.

76. Shih-Lung Lo, op. cit.

77. Idem, p. 223.

78. Idem, p. 230

79. Idem, p. 232.

80. George Sand, Consuelo : La Comtesse de Rodolstadt [1856], tome III, Bibliothèque électronique du Québec, édition en ligne, p. 596-597.

81. Parisis, "La vie parisienne. La Chine à Paris», Le Figaro, 9 mai 1884, p. 1-2 : à propos des inscriptions chinoises sur les objets de la collection de $\mathrm{M}^{\mathrm{me}}$ de Pompadour. 
Figure $n^{\circ} 1^{82}$
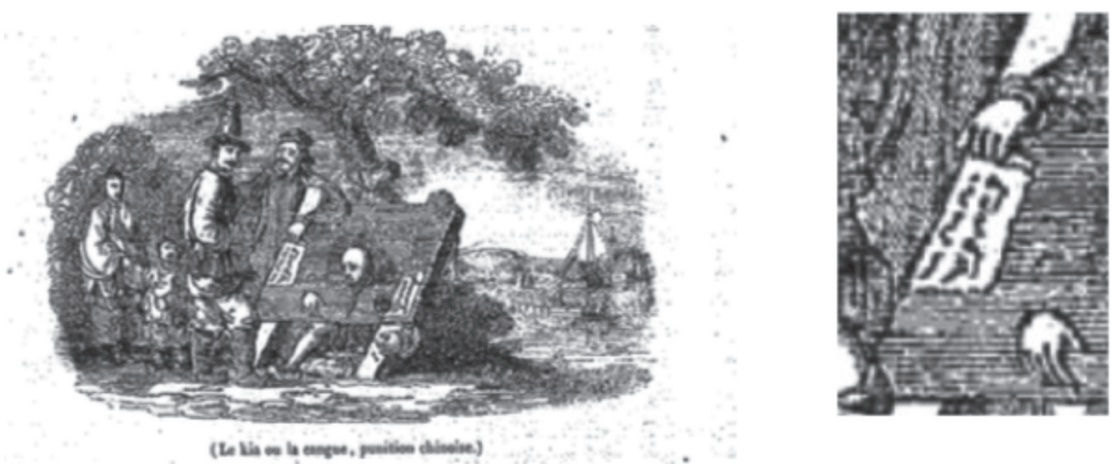

Figure $n^{\circ} 2^{83}$
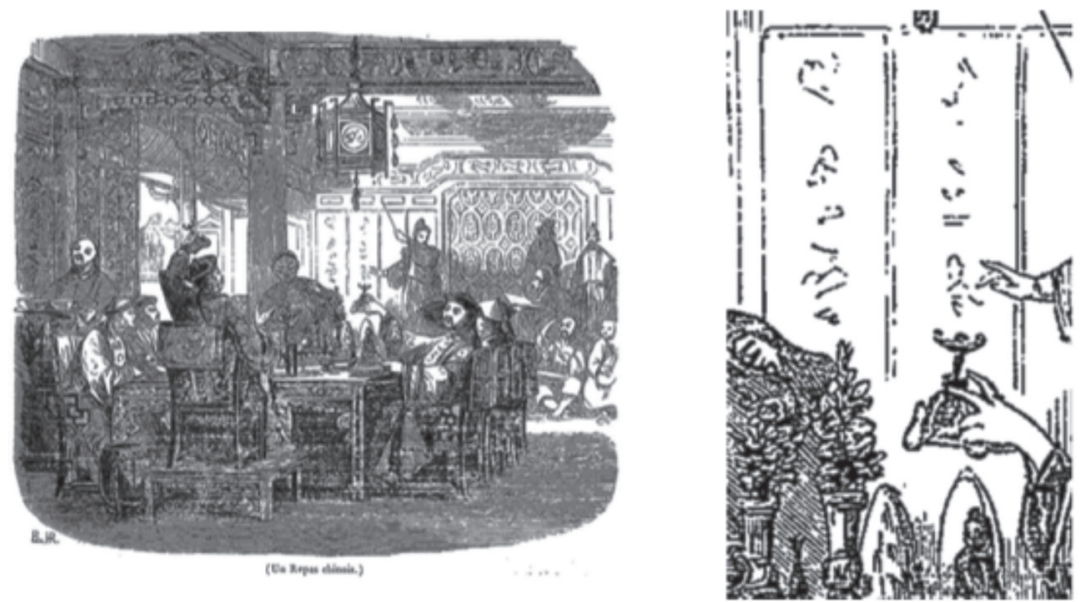

Ainsi, dans le cas des prononciations comme dans celui de l'écriture, c'est l'étrangeté qui, seule, permet d'identifier la langue chinoise.

Il ne faut pas croire toutefois que le Paris profane soit demeuré tout à fait hermétique à l'idée selon laquelle la langue chinoise constitue un objet de connaissances. Cette vision est même largement mobilisée par les représentations profanes, mais sous un angle particulier : la langue chinoise apparaît comme l'objet d'une connaissance incongrue, ce qui ressort de façon manifeste de l'image du sinisant. La maîtrise du chinois apparaît ainsi parfois comme un critère de distinction, comme l'ultime degré du savoir. Un article du Figaro de 1874 nous décrit le Révérend Père Talliez comme "un homme des plus distingués» : «Non-seulement il parle quatre des langues

82. Anonyme, "Code Pénal chinois», Le Magasin Pittoresque, 1839, p. 224 (@ gallica.bnf.fr).

83. Anonyme, "Coutumes chinoises. Le repas chez les riches", Le Magasin Pittoresque, 1844, p. 233 (@) gallica.bnf.fr). 
européennes, mais encore le chinois et l'indien ${ }^{84}$. En 1876, c'est "le petit Woolwich XXXVIII» qui est présenté comme un personnage surprenant, dont l'ultime qualité est la maîtrise de la langue chinoise : «Il est surprenant, le petit Woolwich XXXVIII. Il est noble, élégant, aristocratique. Il sait tout à fond, la grammaire, l'histoire, la géographie, le grec, l'anglais, le latin, l'allemand, le chinois!..." ${ }^{85}$ Quant au comte de Courchamps, il affirme dans le texte qu'il consacre aux Duchesses dans la collection Les Français peints par eux-mêmes, que les bas blancs constituent «le seul rapport qu'il y ait entre cette femme supérieure et les femmes vulgaires, entre une duchesse qui étudie le chinois, et des bourgeoises de Paris qui lisent Paul de Kock» ${ }^{86}$ : la langue chinoise apparaît ainsi comme l'étude la plus opposée qui soit aux lectures vulgaires.

Toutefois, cette connaissance se voit le plus souvent stigmatiser comme pédante et ridicule. L'exemple le plus éloquent de cette satire du sinisant se trouve dans le roman Georges d'Alexandre Dumas. Lorsque Sara raconte à son oncle M. de Malmédie et à son cousin Henri comment le personnage éponyme lui a servi d'interprète avec un Chinois rencontré à Port-Louis, la réaction d'Henri témoigne du ridicule qui frappe alors la connaissance sinologique :

«- Mais ce monsieur parle donc chinois? demanda Henri avec étonnement.

- Oui, répondit Sara.

- Oh! mon père, s'écria Henri en éclatant de rire; oh! vous ne savez pas : il parle chinois!

- Eh bien, qu'y a-t-il de si risible à cela? demanda Sara.

- Oh! rien du tout, reprit Henri en continuant de s'abandonner à son hilarité. Comment donc! mais c'est un charmant talent que possède là le bel étranger, et c'est un homme bien heureux. Il peut causer avec les boîtes à thé et les paravents ${ }^{87}$.

Cette vision de la langue chinoise comme un savoir inutile et ridicule, ne permettant de parler qu'avec les boîtes à thé, ressort aussi logiquement des représentations qui entourent l'enseignement du chinois. La presse brode ainsi inlassablement tout au long du siècle sur le motif du professeur de chinois récitant son "cours drôlatique ${ }^{88}$ devant une salle vide, d'où l'élaboration d'un type récurrent de plaisanterie, que nous pouvons nommer la blague de professeur de chinois. Ce genre, principalement représenté par Pierre Véron, en vient à adopter un schéma immuable : le professeur entre un jour

84. Gaston Vassy, «Informations», Le Figaro, 30 septembre 1874, p. 2.

85. Touchatout, "Le petit prodige Woolwich XXXVIII", Tintamarre, 9 janvier 1876, p. 5.

86. M. le comte de Courchamps, "Les Duchesses», in Collectif, Les Français peints par eux-mêmes. Encyclopédie morale du dix-neuvième siècle, Léon Curmer, 1840-1842, tome premier : "Paris», p. 101. 87. Alexandre Dumas, Georges, Paris, Gallimard, Collection Folio classique, 1974 [1843], p. 224225.

88. Pierre Véron, «Courrier de Paris», Le Monde illustré, 4 avril 1874, p. 211. 
dans sa salle de classe pour y découvrir, ô surprise! d'innombrables auditeurs, s'imagine que son cours suscite enfin l'intérêt, mais découvre finalement que la cause de cette affluence soudaine est tout autre - que ce soit la pluie, une fusillade dans le quartier ou encore, comme dans l'exemple suivant, la curiosité pour un cours aussi grotesque :

"C'était dans les premiers temps de la nomination de M. Stanislas Jullien [sic]. Il faisait son cours les jeudis et samedis. Pendant le premier mois, il ne vit personne, que le poêle, qui ronflait furieusement. Mais un jour, ô stupéfaction! il vit entrer une bande de jolies femmes et de gentlemen bien mis, qui prirent place sur les bancs.

M. Jullien, enchanté, commença son cours, et, pris d'un accès de galanterie, traduisit un sonnet chinois du poète Li-O-Tsing, intitulé : Le Lotus du fleuve jaune. La femme y est comparée à ces jolis petits poissons qu'on appelle Cyprins dorés. L'assistance le regardait curieusement. Le cours fini, M. Jullien sortit enchanté.

Le samedi suivant, autre visite du même genre. M. Jullien reconnut, parmi les nouveaux venus, un de ses auditeurs de l'avant-veille.

- Ah! se dit-il, voilà au moins un homme qui apprécie le chinois!...

Et il traduisit un autre sonnet, celui-là du poète Ma-Tang.

Toutes les semaines, le même monsieur revint, accompagné de différents personnages. Ce ne fut que six mois après que $M$. Jullien apprit que c'était un cicerone, faisant métier de promener les étrangers dans Paris. Une des choses qu'il leur faisait voir comme les plus curieuses, c'était un monsieur d'aspect sérieux, enseignant sérieusement le chinois. ${ }^{89}$

Plus qu'une diffusion du chinois savant vers le monde non-sinologique, cette exploration du chinois profane permet ainsi de dégager une conception originale de la langue chinoise, conçue comme le paradigme même de l'altérité et de la bizarrerie, qui se réapproprie l'image d'une langue chinoise-objet de savoir plus qu'elle ne se laisse véritablement influencer par elle.

L'extrême diversité des conceptions parisiennes de la langue chinoise semble mettre à mal l'idée même d'un unique chinois parisien. Les Parisiens ne sont pas tous confrontés aux mêmes échantillons de langue chinoise - selon qu'ils accèdent ou non aux cours de chinois et aux bibliothèques, selon qu'ils ont ou non voyagé en Chine - et les efforts de vulgarisation entrepris par les sinologues ne semblent pas venir à bout de l'irréductible fossé qui sépare un chinois savant conçu comme une langue originale mais comparable à n'importe quel autre système de communication linguistique,

89. Gaston Vassy, «Informations. La Journée», Le Figaro, 23 mai 1874, p. 3. 
et un chinois profane limitant la langue chinoise à l'impression première d'altérité et d'étrangeté que suscitent sa prononciation comme son écriture.

Il ressort toutefois de cette étude, sinon un chinois parisien clairement défini, du moins un spectre parisien de conceptions de la langue chinoise. Les conceptions parisiennes de cet idiome sont largement tributaire de ce que la ville recèle de chinois et l'image - héritée du XVIII siècle - d'une langue chinoise érigée en paradigme de l'incompréhensibilité, en figure archétypique de l'Autre, demeure l'arrière-plan des différentes représentations qui entourent cet idiome. Que les sinologues cherchent à mesurer l'écart qui sépare le chinois des langues européennes ou que les caricaturistes accentuent cette distance, c'est toujours la question de son altérité qui est au cœur des discours relatifs au chinois. Par ailleurs, chinois savant et chinois profane ne sont pas absolument hermétiques l'un à l'autre. Les sinologues ont conscience du ridicule qui frappe leur objet d'étude, et le Paris profane sait que le chinois dont il rit fait l'objet d'un enseignement - ne serait-ce que grâce aux caricatures qui visent ce dernier. Aussi faut-il considérer les différentes conceptions parisiennes du chinois comme se mouvant, en fonction des motivations des individus, entre les deux pôles de l'altérité risible et de l'objet de science. C'est dans ce tiraillement que réside au XIX ${ }^{\mathrm{e}}$ siècle le chinois parisien.

Clément Fabre est doctorant en histoire contemporaine à l'université Paris 1 Panthéon-Sorbonne. 